\title{
FROBENIUS SPLITTING OF EQUIVARIANT CLOSURES OF REGULAR CONJUGACY CLASSES
}

\author{
JESPER FUNCH THOMSEN
}

\begin{abstract}
Let $G$ denote a connected semisimple and simply connected algebraic group over an algebraically closed field $k$ of positive characteristic and let $g$ denote a regular element of $G$. Let $X$ denote any equivariant embedding of $G$. We prove that the closure of the conjugacy class of $g$ within $X$ is normal and CohenMacaulay. Moreover, when $X$ is smooth we prove that this closure is a local complete intersection. As a consequence, the closure of the unipotent variety within $X$ share the same geometric properties.
\end{abstract}

\section{INTRODUCTION}

Let $G$ denote a connected semisimple linear algebraic group over an algebraically closed field $k$ of positive characteristic. Consider $G$ as a $G \times G$-variety by left and right translation. An equivariant $G$ embedding is a normal $G \times G$-variety $X$ containing a dense open subset $G \times G$-equivariantly isomorphic to $G$. Let $B$ denote a Borel subgroup of $G$ and let $g$ denote an element of $G$. The closure of the double coset $B g B$ within $X$ is called a large Schubert variety. As proved in [2] (see also [1]) large Schubert varieties are normal and Cohen-Macaulay. In the present paper we will prove a similar result for closures of diagonal $G$-orbits of regular elements within $X$.

Assume that $G$ is simply connected. An element $g$ in $G$ is called regular if the centralizer of $g$ in $G$ is of dimension equal to the rank $l$ of $G$. By [11] the set of $G$-conjugacy classes of regular elements is parametrized by the set of points in affine space $\mathbb{A}^{l}$. To describe this correspondence, let $\chi_{1}, \ldots, \chi_{l}$ denote the set of fundamental $G$ characters and consider the Steinberg map $\chi: G \rightarrow \mathbb{A}^{l}$ defined by $\chi(h)=\left(\chi_{1}(h), \ldots, \chi_{l}(h)\right)$. Then the fiber $\chi^{-1}(\mathbf{a})$, associated to a point a in $\mathbb{A}^{l}$, contains a unique open dense $G$-orbit consisting of the set of regular elements within $\chi^{-1}(\mathbf{a})$. Hence, $\chi^{-1}(\mathbf{a})$ is the closure of a unique regular conjugacy class within $G$.

Let $g$ denote any regular element within $G$. We prove that the closure $\overline{C_{G}(g)}$ of the $G$-conjugacy class of $g$ within an equivariant embedding $X$ of $G$ is normal and Cohen-Macaulay. Moreover, when $X$ is smooth we prove that $\overline{C_{G}(g)}$ is a local complete intersection and we calculate its associated dualizing sheaf. When $X=G$ this statement is equivalent to saying that the fibers of the Steinberg map are all normal, 
Cohen-Macaulay and local complete intersections. The latter statement is due to Steinberg (Thm.6.11 and Thm.8.1 in [11]). The case of primary interest to us is when $g$ is a regular unipotent element in $G$. In this case $\overline{C_{G}(g)}$ coincides with the closure of the unipotent variety $\mathcal{U}$ within $X$. In particular, when $G$ coincides with the associated group of adjoint type (i.e. type $E_{8}, F_{4}$ and $G_{2}$ ) we obtain a description of the geometry of the closure of the unipotent variety within the wonderful compactification of $G$.

In order to prove the described results we will use the theory of Frobenius splitting. It is well known that any equivariant embedding $X$ of $G$ is Frobenius split. We prove that there exists a Frobenius splittings of $X$ which compatibly splits the closure $\overline{C_{G}(g)}$ of a given regular conjugacy class. Moreover, we may choose such a splitting to be canonical in the sense of Mathieu (see Chap. 4, [3]). In particular, when $\mathcal{L}$ is a $G$-linearized line bundle on $\overline{C_{G}(g)}$, then the associated set of global sections $\mathrm{H}^{0}\left(\overline{C_{G}(g)}, \mathcal{L}\right)$ admits a good filtration (as a $G$-module). Finally, we prove that when $X$ (and hence $\overline{C_{G}(g)}$ ) is projective then the

higher cohomology groups of globally generated line bundles on $\overline{C_{G}(g)}$ are zero.

\section{Notation}

In the following sections $G$ will denote a connected semisimple linear algebra group over an algebraically closed field $k$ of positive characteristic $p>0$. The associated group of adjoint type will be denoted $G_{\text {ad }}$. We will fix a Borel subgroup $B$ and a maximal torus $T$ within $B$. The character (resp. co-characters) of $T$ will be denoted by $X^{*}(T)$ (resp. $\left.X_{*}(T)\right)$. We identify $X^{*}(T)$ with the group of $B$-characters $X^{*}(B)$.

The set of roots associated with $T$ will be denoted by $R$. We define a root to be positive if the associated $T$-weight space (under the adjoint action) in the Lie algebra of $B$ is zero. The set of positive roots is denoted by $R^{+}$and the associated set of simple roots is denoted by $\Delta=\left\{\alpha_{1}, \ldots, \alpha_{l}\right\}$. To each root $\alpha$ in $R$ there is an associated coroot $\alpha^{\vee}$ which we use to define the set of dominant weights in $X^{*}(T)$. When $G$ is simply connected every dominant weight is a positive linear combination of the fundamental dominant weights $\omega_{1}, \ldots, \omega_{l}$, where $\omega_{i}$ denotes the fundamental weight associated to $\alpha_{i}$.

The Weyl group $W$ associated with $T$ parametrizes the set of Schubert varieties $X(w)$ in the flag variety $G / B$. When $w \in W$ is represented by an element $\dot{w} \in G$ we will write $B w B$ for the subset $B \dot{w} B$ of $G$. The simple reflections, denoted by $s_{1}, \ldots, s_{l}$, generate the Weyl group and we use them to define the length $l(w)$ of an element $w \in W$. The unique element in $W$ of maximal length is denoted by $w_{0}$.

For any $B$-character $\lambda$ we define a $G$-module by

$$
\mathrm{H}(\lambda)=\left\{f: G \rightarrow k: f(g b)=\lambda(b)^{-1} f(g), b \in B, g \in G\right\} .
$$


Then $\mathrm{H}(\lambda)$ is nonzero exactly when $\lambda$ is a dominant $T$-character. When $\mathrm{H}(\lambda)$ is nonzero there exists a unique $B$-stable line within $\mathrm{H}(\lambda)$. This line is called a lowest weight line and its associated weight is $w_{0} \lambda$. Similarly, $H(\lambda)$ contains a unique highest weight line invariant under the opposite Borel group $B^{+}$and of weight $\lambda$. The $G$-module $\mathrm{H}(\lambda)$ coincides with the global sections of a unique $G$-linearized line bundle on $G / B$ which we denote by $\mathcal{L}_{G / B}(\lambda)$.

When $M$ is a $G$-module we consider the group of endomorphisms $\operatorname{End}(M)$ as a $G \times G$-module by

$$
\left(\left(g^{\prime}, g\right) \cdot f\right)(m)=g^{\prime} \cdot\left(f\left(g^{-1} \cdot m\right)\right)
$$

for $g, g^{\prime} \in G, f \in \operatorname{End}(M)$ and $m \in M$. When considering $\operatorname{End}(M)$ as a $G$-module we do this by identifying $G$ with the subgroup $G \times\{e\}$ of $G \times$ $G$. The field $k$ will always be considered as the trivial representation.

2.1. The Steinberg map. Let now $G$ denote a simply connected group and let $M$ denote a finite dimensional rational $G$-module defined by a morphism : $G \rightarrow \mathrm{GL}(\mathrm{M})$. The $G$-character $\chi_{M}: G \rightarrow k$ of $M$ is by definition the composition of latter map with the trace function on GL(M). The Steinberg map ([1] ) is the map

$$
\begin{gathered}
\chi: G \rightarrow \mathbb{A}^{l}, \\
g \mapsto\left(\chi_{1}(g), \ldots, \chi_{l}(g)\right),
\end{gathered}
$$

defined as the product of the $G$-characters $\chi_{i}$ associated to the $G$ modules $\mathrm{H}\left(\omega_{i}\right)$. When $\mathbf{a}=\left(a_{1}, \ldots, a_{l}\right) \in \mathbb{A}^{l}$ we will by $\chi^{-1}(\mathbf{a})$ denote the (scheme theoretic) fiber of $\chi$ at the point a. As mentioned in the introduction we may consider the fibers of the Steinberg map as closures of conjugacy classes of regular elements within $G$. Among all fibers the fiber at $\mathbf{a}=\left(\chi_{1}(e), \ldots, \chi_{l}(e)\right)$ is of particular importance as this fiber coincides with the set of unipotent elements within $G$.

\section{Equivariant EMBEDDings}

In this section $G$ denotes a connected semisimple linear algebraic group. We think of $G$ as a $G \times G$-variety by left and right translation. An equivariant $G$-embedding (or simply a $G$-embedding) is a normal $G \times G$-variety $X$ containing an open subset which is $G \times G$-equivariantly isomorphic to $G$.

3.1. The wonderful compactification. When $G=G_{\text {ad }}$ is of adjoint type there exists a distinguished equivariant embedding $\mathbf{X}$ of $G$ which is called the wonderful compactification (see e.g. Section 6.1, 3]).

The wonderful compactification $\mathbf{X}$ is a smooth projective variety such that the complement of the open subset $G$ is a finite union of smooth irreducible divisors which intersect transversally. Moreover, the common intersection of the irreducible components of $\mathbf{X} \backslash G$ is a closed $G \times G$-orbit $\mathbf{Y}$ which is $G \times G$-equivariantly isomorphic to 
$G / B \times G / B$. The variety $\mathbf{Y}$ is the unique closed $G \times G$-orbit within $X$.

3.2. Toroidal embeddings. An embedding $X$ of a semisimple group $G$ is called toroidal if the canonical map $\phi: G \rightarrow G_{\mathrm{ad}}$ admits an extension $\pi_{X}: X \rightarrow \mathbf{X}$ into the wonderful compactification $\mathbf{X}$ of the group $G_{\text {ad }}$ of adjoint type.

When $X$ is a complete toroidal embedding then every closed orbit of $X$ will map to the unique closed orbit $\mathbf{Y} \simeq G / B \times G / B$ within $\mathbf{X}$. As a consequence, every closed orbit of $X$ is then $G \times G$-equivariantly isomorphic to $G / B \times G / B$.

A toroidal embedding is uniquely determined by the closure $\bar{T}$ of $T$ within $X$. The closure $\bar{T}$ is normal and hence $\bar{T}$ is a toric variety with respect to $T$. As toric varieties admits resolutions by smooth toric varieties this essentially explains the following (see also Prop.6.2.5, [3]

Theorem 3.1. For any equivariant $G$-embedding $X$ there exists a smooth toroidal embedding $X^{\prime}$ of $G$ and a birational projective morphism $X^{\prime} \rightarrow X$ extending the identity map on $G$.

\section{Frobenius Splitting}

Let $X$ denote a scheme of finite type over an algebraically closed field $k$ of characteristic $p>0$. The absolute Frobenius morphism on $X$ is the morphism $F: X \rightarrow X$ of schemes, which is the identity on the set of points and where the associated map of sheaves

$$
F^{\sharp}: \mathcal{O}_{X} \rightarrow F_{*} \mathcal{O}_{X}
$$

is the $p$-th power map. We say that $X$ is Frobenius split if there exists a morphism $s \in \operatorname{Hom}_{\mathcal{O}_{X}}\left(F_{*} \mathcal{O}_{X}, \mathcal{O}_{X}\right)$ such that the composition $s \circ F^{\sharp}$ is the identity map on $\mathcal{O}_{X}$.

4.1. Stable Frobenius splittings along divisors. Let $D$ denote an effective Cartier divisor on $X$ with associated line bundle $\mathcal{O}_{X}(D)$ and canonical section $\sigma_{D}$. We say that $X$ is stably Frobenius split along $D$ if there exists a positive integer $e$ and an morphism

$$
s \in \operatorname{Hom}_{\mathcal{O}_{X}}\left(F_{*}^{e} \mathcal{O}_{X}(D), \mathcal{O}_{X}\right),
$$

such that $s\left(\sigma_{D}\right)=1$. In this case we say that $s$ is a stable Frobenius splitting of $X$ along $D$ of degree $e$. Notice that $X$ is Frobenius split exactly when there exists a stable Frobenius splitting of $X$ along the zero divisor $D=0$.

Remark 1. Consider an element $s \in \operatorname{Hom}_{\mathcal{O}_{X}}\left(F_{*}^{e} \mathcal{O}_{X}(D), \mathcal{O}_{X}\right)$. Then the condition $s\left(\sigma_{D}\right)=1$ on $s$ for it to define a stable Frobenius splitting of $X$, may be checked on any open dense subset of $X$. 
4.2. Subdivisors. Let $D^{\prime} \leq D$ denote an effective Cartier subdivisor and let $s$ be a stable Frobenius splitting of $X$ along $D$ of degree $e$. The composition of $s$ with the map

$$
F_{*}^{e} \mathcal{O}_{X}\left(D^{\prime}\right) \rightarrow F_{*}^{e} \mathcal{O}_{X}(D),
$$

defined by the canonical section of the divisor $D-D^{\prime}$, is then a stable Frobenius splitting of $X$ along $D^{\prime}$ of degree $e$. Applying this to the case $D^{\prime}=0$ it follows that if $X$ is stably Frobenius split along any effective divisor $D$ then $X$ is also Frobenius split.

Lemma 4.1. Let $D_{1}$ and $D_{2}$ denote effective Cartier divisors. If $s_{1}$ (resp. $s_{2}$ ) is a stable Frobenius splitting of $X$ along $D_{1}$ (resp. $D_{2}$ ) of degree $e_{1}$ (resp. $\left.e_{2}\right)$, then there exists a stable Frobenius splitting of $X$ along $D_{1}+D_{2}$ of degree $e_{1}+e_{2}$.

Proof. By the discussion above it suffices to prove that there exists a stable Frobenius splitting of $X$ along $D_{1}+p^{e_{1}} D_{2}$ of degree $e_{1}+e_{2}$. By the projection formula there exists an identification

$$
F_{*}^{e_{1}+e_{2}} \mathcal{O}_{X}\left(D_{1}+p^{e_{1}} D_{2}\right) \simeq F_{*}^{e_{2}}\left(\mathcal{O}_{X}\left(D_{2}\right) \otimes F_{*}^{e_{1}} \mathcal{O}_{X}\left(D_{1}\right)\right) .
$$

where we have used the relation $F^{*} O_{X}\left(D_{2}\right) \simeq O_{X}\left(p D_{2}\right)$. Hence, $s_{1}$ defines a map

$$
F_{*}^{e_{1}+e_{2}} \mathcal{O}_{X}\left(D_{1}+p^{e_{1}} D_{2}\right) \rightarrow F_{*}^{e_{2}}\left(\mathcal{O}_{X}\left(D_{2}\right)\right) .
$$

Composing the latter map with $s_{2}$ we obtain a map

$$
F_{*}^{e_{1}+e_{2}} \mathcal{O}_{X}\left(D_{1}+p^{e_{1}} D_{2}\right) \rightarrow \mathcal{O}_{X},
$$

which defines the desired stable Frobenius splitting of $X$.

4.3. Compatibly split subschemes. Let $Y$ denote a closed subscheme of $X$ with sheaf of ideals $\mathcal{I}_{Y}$. When

$$
s \in \operatorname{Hom}_{\mathcal{O}_{X}}\left(F_{*}^{e} \mathcal{O}_{X}(D), \mathcal{O}_{X}\right),
$$

is a stable Frobenius splitting of $X$ along $D$ we say that $s$ compatibly Frobenius splits $Y$ if the following conditions are satisfied

(1) The support of $D$ does not contain any of the irreducible components of $Y$.

(2) $s\left(F_{*}^{e}\left(\mathcal{I}_{Y} \otimes \mathcal{O}_{X}(D)\right)\right) \subseteq \mathcal{I}_{Y}$.

When $s$ compatibly Frobenius splits $Y$ there exists an induced stable Frobenius splitting of $Y$ along $D \cap Y$ of degree $e$. Notice that when only condition (2) is satisfied then $Y$ is still compatibly Frobenius split by the induced stable Frobenius splitting of $X$ along the zero divisor 0 . In concrete situations condition (2) may be checked using the following result

Lemma 4.2. Let $s$ denote a stable Frobenius splitting of $X$ along a divisor $D$ and let $Y$ denote a closed subscheme of $X$ satisfying the above condition (1). If $Y$ is compatibly Frobenius split by the induced 
stable Frobenius splitting of $X$ along the zero divisor $0 \leq D$, then $Y$ is also compatibly Frobenius split by s.

Proof. Argue as in the proof of Prop.1.4 in [9].

Lemma 4.3. Let $s$ denote a stable Frobenius splitting of $X$ along $D$ which compatibly Frobenius splits a closed subscheme $Y$ of $X$. If $D^{\prime} \leq$ $D$ then the induced stable Frobenius splitting of $X$ along $D^{\prime}$, defined in Section 4.2. compatibly Frobenius splits $Y$.

Proof. This follows immediately from the construction of the induced Frobenius splitting of $X$ along $D^{\prime}$.

Lemma 4.4. Let $D_{1}$ and $D_{2}$ denote effective Cartier divisors. If $s_{1}$ (resp. $s_{2}$ ) is a stable Frobenius splitting of $X$ along $D_{1}$ (resp. $D_{2}$ ) of degree $e_{1}$ (resp. $e_{2}$ ) which compatibly splits a closed subscheme $Y$ of $X$, then there exists a stable Frobenius splitting of $X$ along $D_{1}+D_{2}$ of degree $e_{1}+e_{2}$ which compatibly splits $Y$.

Proof. The stable Frobenius splitting of $X$ along $D_{1}+D_{2}$ defined in the proof of Lemma 4.1 compatibly Frobenius splits $Y$.

As an easy consequence of the above definitions we find

Lemma 4.5. Let $s$ denote a stable Frobenius splitting of $X$ along an effective divisor $D$. Then

(1) $X$ is reduced and every irreducible component of $X$ is compatibly Frobenius split.

(2) If s compatibly Frobenius splits a closed subscheme $Y$ of $X$ then each irreducible component of $Y$ is also compatibly Frobenius split by $s$.

(3) Assume that s compatibly Frobenius splits closed subschemes $Y_{1}$ and $Y_{2}$ and that the support of $D$ does not contain any of the irreducible components of the scheme theoretic intersection $Y_{1} \cap Y_{2}$. Then s compatibly Frobenius splits $Y_{1} \cap Y_{2}$.

The following statement relates stable Frobenius splitting along divisors with compatibly Frobenius splitting.

Lemma 4.6. Let $D$ and $D^{\prime}$ denote effective Cartier divisors and let $s$ denote a stable Frobenius splitting of $X$ along $(p-1) D+D^{\prime}$ of degree 1. Then there exists a stable Frobenius splitting of $X$ along $D^{\prime}$ of degree 1 which compatibly splits the closed subscheme defined by D.

Proof. Let $s$ denote a stable Frobenius splitting along $(p-1) D+D^{\prime}$ of degree 1 . Define $s^{\prime}$ to be the composition of $s$ with the map

$$
F_{*} \mathcal{O}_{X}\left(D^{\prime}\right) \rightarrow F_{*} \mathcal{O}_{X}\left((p-1) D+D^{\prime}\right),
$$

defined by the canonical section $\sigma_{D}^{p-1}$ of $(p-1) D$. Then $s^{\prime}$ is a stable Frobenius splitting of $X$ of degree 1. It remains to show that $D$ is compatibly Frobenius split by $s^{\prime}$. As this is a local condition we may 
assume that $\mathcal{O}_{X}(D)$ and $\mathcal{O}_{X}\left(D^{\prime}\right)$ are trivial line bundles and that $X$ is affine. Identify $\sigma_{D}$ and $\sigma_{D^{\prime}}$ with elements in the coordinate ring $k[X]$ and consider $s$ and $s^{\prime}$ as maps from $F_{*} k[X]$ to $k[X]$. By definition

$$
s^{\prime}(a)=s\left(a \sigma_{D}^{p-1}\right), a \in k[X] .
$$

Hence, when $a=\sigma_{D} a^{\prime} \in\left(\sigma_{D}\right)$ belongs to the ideal generated by $\sigma_{D}$ we find

$$
s^{\prime}(a)=s\left(\sigma_{D}^{p} a^{\prime}\right)=\sigma_{D} s\left(a^{\prime}\right) \in\left(\sigma_{D}\right) .
$$

It remains to prove that none of the components of $D$ is contained in the support of $D^{\prime}$. Restricting, if necessary, to an open subset we may assume that $D$ is irreducible and nonempty. Now assume that $D$ is contained in the support of $D^{\prime}$. By Lemma 4.5 the ideal $\left(\sigma_{D}\right)$ is radical. Hence, $\sigma_{D^{\prime}}$ is contained in $\left(\sigma_{D}\right)$. In particular,

$$
1=s^{\prime}\left(\sigma_{D^{\prime}}\right) \in\left(\sigma_{D}\right)
$$

which is a contradiction.

4.4. Cohomology and Frobenius splitting. The notion of Frobenius splitting is particular useful in connection with proving higher cohomology vanishing for line bundles. The main idea is that when $s \in \operatorname{Hom}_{\mathcal{O}_{X}}\left(F_{*}^{e} \mathcal{O}_{X}(D), \mathcal{O}_{X}\right)$ is a stable Frobenius splitting of $X$ along the divisor $D$, then $s$ defines a splitting of the injective map

$$
\mathcal{O}_{X} \rightarrow F_{*}^{e} \mathcal{O}_{X}(D) \text {. }
$$

Tensoring the latter map with a line bundle $\mathcal{L}$ on $X$ we find a split injective map

$$
\mathcal{L} \rightarrow F_{*}^{e}\left(\mathcal{L}^{p^{e}} \otimes \mathcal{O}_{X}(D)\right),
$$

where we have applied the projection formula and the relation $F^{*} \mathcal{L}=$ $\mathcal{L}^{p}$. As $F$ is a finite morphism the following statement is an easy consequence.

Lemma 4.7. Let $s$ denote a stable Frobenius splitting of $X$ along $D$ of degree $e$. Then for every line bundle $\mathcal{L}$ on $X$ and every integer $i$ there exists an inclusion

$$
\mathrm{H}^{i}(X, \mathcal{L}) \subseteq \mathrm{H}^{i}\left(X, \mathcal{L}^{p^{e}} \otimes \mathcal{O}_{X}(D)\right),
$$

of abelian groups. In particular, when $X$ is projective, $\mathcal{L}$ is globally generated and $D$ is ample then the group $\mathrm{H}^{i}(X, \mathcal{L})$ is zero for $i>0$.

Assume now, moreover, that $Y$ is compatibly Frobenius split by the stable Frobenius splitting $s$ of $X$. Then $s$ defines a splitting of the map

$$
\begin{gathered}
\mathcal{I}_{Y} \rightarrow F_{*}^{e}\left(\mathcal{I}_{Y} \otimes \mathcal{O}_{X}(D)\right), \\
f \mapsto f^{p^{e}} \sigma_{D} .
\end{gathered}
$$

Tensoring the latter map with a line bundle $\mathcal{L}$ on $X$ then leads to 
Lemma 4.8. Let $s$ denote a stable Frobenius splitting of $X$ along $D$ of degree $e$ and let $Y$ denote a closed compatibly Frobenius split subscheme of $X$. Then for every line bundle $\mathcal{L}$ on $X$ and every integer $i$ there exists an inclusion

$$
\mathrm{H}^{i}\left(X, \mathcal{I}_{Y} \otimes \mathcal{L}\right) \subseteq \mathrm{H}^{i}\left(X, \mathcal{I}_{Y} \otimes \mathcal{L}^{p^{e}} \otimes \mathcal{O}_{X}(D)\right),
$$

of abelian groups. In particular, when $X$ is projective, $\mathcal{L}$ is globally generated and $D$ is ample then the group $\mathrm{H}^{i}\left(X, \mathcal{I}_{Y} \otimes \mathcal{L}\right)$ is zero for $i>0$.

4.5. Push forward. Let $f: X \rightarrow X^{\prime}$ denote proper morphism of schemes and assume that the induced map $\mathcal{O}_{X^{\prime}} \rightarrow f_{*} \mathcal{O}_{X}$ is an isomorphism. Then every Frobenius splitting of $X$ induces, by application of the functor $f_{*}$, a Frobenius splitting of $X^{\prime}$. Moreover, when $Y$ is a compatibly Frobenius split subscheme of $X$ then the induced Frobenius splitting of $X^{\prime}$ compatibly splits the scheme theoretic image $f(Y)$ (Prop.4, 6]).

We will need the following connected statement.

Lemma 4.9. Let $f: X \rightarrow X^{\prime}$ denote a morphism of projective schemes such that $\mathcal{O}_{X^{\prime}} \rightarrow f_{*} \mathcal{O}_{X}$ is an isomorphism. Let $Y$ be a closed subscheme of $X$ and denote by $Y^{\prime}$ the scheme theoretic image $f(Y)$. Assume that there exists a stable Frobenius splitting of $X$ along an ample divisor $D$ which compatibly splits $Y$. Then $f_{*} \mathcal{O}_{Y}=\mathcal{O}_{Y^{\prime}}$ and $R^{i} f_{*} \mathcal{O}_{Y}=0$ for $i>0$.

Proof. Let $\mathcal{L}$ denote an ample line bundle on $X^{\prime}$. By a result of $\mathrm{G}$. Kempf (see Lemma 2.11, 9]) it suffices to prove, for sufficiently large values of $n$, that

(1) $\mathrm{H}^{i}\left(X, f^{*} \mathcal{L}^{n}\right)=\mathrm{H}^{i}\left(Y, f^{*} \mathcal{L}^{n}\right)=0$ for $i>0$.

(2) The restriction map $\mathrm{H}^{0}\left(X, f^{*} \mathcal{L}^{n}\right) \rightarrow \mathrm{H}^{0}\left(Y, f^{*} \mathcal{L}^{n}\right)$ is surjective.

Now apply the "in particular" statements in Lemma 4.7 and 4.8

4.6. Frobenius splitting of smooth varieties. When $X$ is smooth there exists a canonical $\mathcal{O}_{X}$-linear identification (see e.g. Section 1.3.7 in [3])

$$
C_{X}: F_{*} \omega_{X}^{1-p} \simeq \operatorname{Hom}_{\mathcal{O}_{X}}\left(F_{*} \mathcal{O}_{X}, \mathcal{O}_{X}\right) .
$$

Hence, a Frobenius splitting of $X$ may be identified with a global section of $\omega_{X}^{1-p}$ with certain properties. A global section $s$ of $\omega_{X}^{1-p}$ which corresponds to a Frobenius splitting will be called a Frobenius splitting.

Lemma 4.10. Assume that $X$ is smooth and let $\tau$ denote a global section of $\omega_{X}^{1-p}$ which defines a Frobenius splitting of $X$. Then there exists a stable Frobenius splitting of $X$ of degree 1 along the Cartier divisor defined by $\tau$. In particular, if $\tau=\tilde{\tau}^{p-1}$ is a $(p-1)$-th power of a global section $\tilde{\tau}$ of $\omega_{X}^{-1}$, then $X$ is Frobenius split compatibly with the zero divisor of $\tilde{\tau}$. 
Proof. Composing the evaluation map

$$
\begin{aligned}
\operatorname{Hom}_{\mathcal{O}_{X}}\left(F_{*} \mathcal{O}_{X}, \mathcal{O}_{X}\right) \rightarrow \mathcal{O}_{X}, \\
s \mapsto s(1),
\end{aligned}
$$

with $C_{X}$ defines a stable Frobenius splitting of $X$ of degree 1 along the Cartier divisor defined by $\tau$. This proves the first assertion. The second assertion follows from Lemma 4.6 with $D^{\prime}=0$.

4.7. Frobenius splitting of $G / B$. Let $G$ denote a simply connected linear algebraic group. The flag variety $X=G / B$ is a smooth variety with dual canonical bundle

$$
\omega_{X}^{-1}=\mathcal{L}_{G / B}(2 \rho)
$$

where $\rho$ denotes the sum of the fundamental dominant weights. Consider the multiplication map

$$
\phi_{G / B}: \mathrm{St} \otimes \mathrm{St} \rightarrow \mathrm{H}(2(p-1) \rho)=\mathrm{H}^{0}\left(G / B, \omega_{G / B}^{1-p}\right),
$$

where we have used the notation St to denote the Steinberg module $\mathrm{H}((p-1) \rho)$. The Steinberg module St is an irreducible selfdual $G$ module and hence there exists a unique (up to nonzero scalars) nondegenerate $G$-invariant bilinear form (, ) on St. Now we have the following description

Theorem 4.1. ([5]) Let $h=\sum_{i} f_{i} \otimes g_{i}$ denote an element in St $\otimes$ St. Then $\phi_{G / B}(h)$ defines a Frobenius splitting of $X$ (up to a nonzero scalar) if and only if $\sum_{i}\left(f_{i}, g_{i}\right) \neq 0$.

As an easy consequence of this result (with $G$ substituted with $G \times G$ ) we have

Corollary 4.1. (5) Let $v_{\Delta}$ denote a generator of the unique diagonal $G$-invariant line in $\mathrm{St} \otimes \mathrm{St}$ and let $v_{-}$(resp. $v_{+}$) denote a generator of the highest (resp. lowest) weight line in St. Then

$$
\phi_{(G \times G) /(B \times B)}\left(v_{\Delta} \otimes\left(v_{-} \otimes v_{+}\right)\right)
$$

defines a Frobenius splitting of $G / B \times G / B$ (up to a nonzero scalar).

\section{Preliminary Results}

Throughout this section $G$ is simply connected and $\mu$ is a nonzero fixed dominant $T$-character. 
5.1. The morphism $\psi_{\mu}$. Consider the $G \times G$-equivariant defined by

$$
\begin{aligned}
& \psi_{\mu}: G \rightarrow \mathbb{P}_{\mu}:=\mathbb{P}(\operatorname{End}(\mathrm{H}(\mu)) \oplus k), \\
& g \mapsto\left[\left(g \cdot I_{\mathrm{H}(\mu)}, 1\right)\right],
\end{aligned}
$$

where $I_{\mathrm{H}(\mu)}$ denotes the identity map on $\mathrm{H}(\mu)$. Let $\mathcal{O}_{\mu}(1)$ denote the ample generator of the Picard group of $\mathbb{P}_{\mu}$. The pull back $\psi_{\mu}^{*}\left(\mathcal{O}_{\mu}(1)\right)$ is then (by the description of $\psi_{\mu}$ ) canonical isomorphic to the trivial line bundle on $G$. The induced map on global sections

$$
\psi_{\mu, G}^{*}: \operatorname{End}(\mathrm{H}(\mu))^{*} \oplus k \simeq \mathrm{H}^{0}\left(\mathbb{P}_{\mu}, \mathcal{O}_{\mu}(1)\right) \rightarrow \mathrm{H}^{0}\left(G, \psi_{\mu}^{*}\left(\mathcal{O}_{\mu}(1)\right)\right) \simeq k[G],
$$

is given by

$$
\psi_{\mu, G}^{*}\left(v^{*} \otimes v, a\right)(g)=v^{*}(g v)+a,
$$

with $v^{*} \otimes v \in \mathrm{H}(\mu)^{*} \otimes \mathrm{H}(\mu) \simeq \operatorname{End}(\mathrm{H}(\mu))^{*}$ and $a \in k$.

Let $v_{\mu}^{-}$(resp. $u_{\mu}^{-}$) denote a lowest weight vector in $\mathrm{H}(\mu)$ (resp. $\left.\mathrm{H}(\mu)^{*}\right)$. Similarly we let $v_{\mu}^{+}$(resp. $u_{\mu}^{+}$) denote a highest weight vector in $\mathrm{H}(\mu)$ (resp. $\left.\mathrm{H}(\mu)^{*}\right)$. Let $\operatorname{Tr}_{\mu}$ denote the trace function on $\operatorname{End}(\mathrm{H}(\mu))$. Then

Lemma 5.1. The function $\psi_{\mu, G}^{*}\left(u_{\mu}^{-} \otimes v_{\mu}^{-}, 0\right)$ on $G$ is a generator of the lowest weight space in $\mathrm{H}\left(-w_{0} \mu\right) \subseteq k[G]$. Moreover, the function $\psi_{\mu, G}^{*}\left(\operatorname{Tr}_{\mu}, 0\right)$ coincides with the $G$-character $\chi_{\mu}$ of $\mathrm{H}(\mu)$.

Proof. That $\psi_{\mu, G}^{*}\left(u_{\mu}^{-} \otimes v_{\mu}^{-}, 0\right)$ is nonzero follows by evaluating it at $w_{0}$. The first assertion then follows as $u_{\mu}^{-} \otimes v_{\mu}^{-}$is $B \times B$-semiinvariant (i.e. invariant up to scalars) of weight $\left(-\mu, w_{0} \mu\right)$. The second assertion is clear from the discussion above.

5.1.1. Regular functions on $G$. In connection with Lemma 5.1 we will later need a more precise description of $\psi_{\mu, G}^{*}\left(u_{\mu}^{-} \otimes v_{\mu}^{-}, 0\right)$ when $\mu$ is a fundamental dominant weight. Recall that the coordinate ring $k[G]$ is a unique factorization domain as $G$ is assumed to be simply connected. Then

Lemma 5.2. Let $f_{i}$ denote a generator of the lowest weight space in $\mathrm{H}\left(\omega_{i}\right)$. Then $f_{i}$ is an irreducible element in $k[G]$ and the ideal $\left(f_{i}\right)$ generated by $f_{i}$ in $k[G]$ coincides with the ideal of functions vanishing on the closure $\overline{B w_{0} s_{i} B}$ of the Bruhat cell B $w_{0} s_{i} B$.

Proof. Write $f_{i}$ as a product of irreducible elements $f_{i}=f_{i, 1} \cdots f_{i, n}$. As $f_{i}$ is $B \times B$-semiinvariant each factor $f_{i, j}$ of this product is also $B \times B$-semiinvariant. Hence, there exists dominant weights $\omega_{i, j}$ such that $f_{i, j}$ generates the lowest weight space in $\mathrm{H}\left(\omega_{i, j}\right)$. Moreover, by the factorization of $f_{i}$ we must have $\omega_{i}=\sum_{j=1}^{n} \omega_{i, j}$. But then $n$ must be equal to 1 which proves the first part of the statement.

As $f_{i}$ is irreducible the ideal $\left(f_{i}\right)$ is a prime ideal of height 1 . By the $B \times B$-semiinvariance of $f_{i}$ this ideal must coincide with the ideal of functions vanishing on the closure of some Bruhat cell $\overline{B w_{0} s_{j} B}$ of 
codimension 1. It remains to prove that $f(w)=0$, where $w \in G$ denotes a representative for $w_{0} s_{i}$. So assume that $f(w) \neq 0$. When $t \in T$ we may calculate $f(t w)$ in two different ways. First by definition of $\mathrm{H}\left(\omega_{i}\right)$ :

$$
f(t w)=f\left(w w^{-1} t w\right)=\omega_{i}\left(w^{-1} t^{-1} w\right) f(w)=\left(-w_{0} s_{i} \omega_{i}\right)(t) f(w) .
$$

But also $f(t w)=\left(t^{-1} \cdot f\right)(w)=\left(-w_{0} \omega_{i}\right)(t) f(w)$. In particular, we conclude that $w_{0} s_{i} \omega_{i}=w_{0} \omega_{i}$ which is a contradiction.

5.2. The closed orbit in $Z_{\mu}$. In the sequel we let $Z_{\mu}$ denote the closure of the image of $\psi_{\mu}$.

Lemma 5.3. The element $\left[\left(v_{\mu}^{-} \otimes u_{\mu}^{-}, 0\right)\right]$ is the unique $B \times B$-invariant point of $Z_{\mu}$. In particular, $Z_{\mu}$ contains a unique closed $G \times G$-orbit.

Proof. By Borel's fixed point theorem the set of $B \times B$-invariant points in $Z_{\mu}$ is nonempty. So consider a $B \times B$-invariant point $x=[(f, a)]$, $f \in \operatorname{End}(\mathrm{H}(\mu)), a \in k$, of $Z_{\mu}$. Then $f$ is a $B \times B$-semiinvariant element of $\operatorname{End}(\mathrm{H}(\mu))=\mathrm{H}(\mu) \otimes \mathrm{H}(\mu)^{*}$. In particular, $f$ is a multiple of $v_{\mu}^{-} \otimes u_{\mu}^{-}$. As $v_{\mu}^{-} \otimes u_{\mu}^{-}$is a weight vector of nonzero weight this leaves us with two cases; either $x=\left[\left(v_{\mu}^{-} \otimes u_{\mu}^{-}, 0\right)\right]$ or $x=[(0,1)]$. Now the following Lemma 5.4 excludes the latter case and ends the proof of the first assertion. The second assertion now follows as every closed orbit contains a $B \times B$ invariant point.

Lemma 5.4. Let $M$ denote a finite dimensional $G$-module. The closure in $\mathbb{P}(\operatorname{End}(M) \oplus k)$ of the $G \times G$-orbit through the point $\left[\left(I_{M}, 1\right)\right]$ does not contain the point $[(0,1)]$.

Proof. Let $x$ denote the point $\left[\left(I_{M}, 1\right)\right]$. Then by Lem.6.1.4 in [3]

$$
\overline{(G \times G) \cdot x}=(G \times G) \overline{(T \times T) \cdot x} .
$$

In particular, it is enough to prove that $[(0,1)]$ is not contained in $\overline{(T \times T) \cdot x}=\overline{T \cdot x}$. Choose a basis $m_{i}, i \in I$, of $M$ consisting of $T$ eigenvectors and let $m_{i}^{*}$ denote the dual basis. Then, $m_{i} \otimes m_{j}^{*}, i, j \in I$, is a basis of $\operatorname{End}(M)$. Now adjoin $z=(0,1) \in \operatorname{End}(M) \oplus k$ to obtain a basis for $\operatorname{End}(M) \oplus k$ and let $z^{*}, m_{i}^{*} \otimes m_{j}, i, j \in I$, denote the dual basis. The top wedge product of $M$ is a 1-dimensional $G$-representation and hence it must be the trivial representation. In particular, the homogeneous polynomial function

$$
\left(\prod_{i \in I} m_{i}^{*} \otimes m_{i}\right)-\left(z^{*}\right)^{\operatorname{dim}_{k}(N)}
$$

vanishes on $\overline{T \cdot x}$. Hence, $[(0,1)]$ is not contained in $\overline{T \cdot x}$. 
5.3. Morphisms into $Z_{\mu}$. In order to state the next result we define $p: \operatorname{End}(\mathrm{H}(\mu))^{*} \oplus k \rightarrow \operatorname{End}(\mathrm{H}(\mu))^{*}$ to denote the projection onto the first summand. Recall that by Frobenius reciprocity there exists a unique (up to scalar) $G$-equivariant morphism $\mathrm{H}(\mu)^{*} \rightarrow \mathrm{H}\left(-w_{0} \mu\right)$.

Lemma 5.5. Let $\eta: G / B \times G / B \rightarrow Z_{\mu} \subseteq \mathbb{P}_{\mu}$ denote a $G \times G$ equivariant morphism. Then

$$
\eta^{*}\left(\mathcal{O}_{\mu}(1)\right) \simeq \mathcal{L}_{G / B}\left(-w_{0} \mu\right) \otimes \mathcal{L}_{G / B}(\mu)
$$

and the associated map of global sections $\mathrm{R}_{\mu}$ fits into a commutative diagram



where the right vertical arrow is defined from a (unique up to nonzero scalars) nonzero map $\mathrm{H}(\mu)^{*} \rightarrow \mathrm{H}\left(-w_{0} \mu\right)$ of $G$-modules.

Proof. By Lemma 5.3 the morphism $\eta$ must be given by

$$
\begin{aligned}
\eta: G / B \times G / B & \rightarrow Z_{\mu} \subseteq \mathbb{P}(\operatorname{End}(\mathrm{H}(\mu)) \oplus k), \\
\left(g B, g^{\prime} B\right) & \mapsto\left[\left(g v_{\mu}^{-} \otimes g^{\prime} u_{\mu}^{-}, 0\right)\right] .
\end{aligned}
$$

Hence we may, via the Segre embedding, factor $\eta$ through the map

$$
\begin{aligned}
G / B \times G / B & \rightarrow \mathbb{P}(\mathrm{H}(\mu)) \times \mathbb{P}\left(\mathrm{H}(\mu)^{*}\right), \\
\left(g B, g^{\prime} B\right) & \mapsto\left(\left[\left(g \cdot v_{\mu}^{-}\right],\left[g^{\prime} \cdot u_{\mu}^{-}\right]\right) .\right.
\end{aligned}
$$

As a consequence, we find that

$$
\eta^{*}\left(\mathcal{O}_{\mu}(1)\right) \simeq \mathcal{L}_{G / B}\left(-w_{0} \mu\right) \otimes \mathcal{L}_{G / B}(\mu) .
$$

Moreover the associated map on global sections

$$
\mathrm{R}_{\mu}: \operatorname{End}(\mathrm{H}(\mu))^{*} \oplus k=\mathcal{O}_{\mu}(1)\left(\mathbb{P}_{\mu}\right) \rightarrow \mathrm{H}\left(-w_{0} \mu\right) \otimes \mathrm{H}(\mu)
$$

is nonzero and factorizes through the projection map $p$.

Lemma 5.6. The element $R_{\mu}\left(u_{\mu}^{+} \otimes v_{\mu}^{-}, 0\right)$ is a nonzero multiple of $v_{-w_{0} \mu}^{+} \otimes v_{\mu}^{-}$. Moreover, the element $R_{\mu}\left(\operatorname{Tr}_{\mu}, 0\right)$ is a nonzero generator of the unique diagonal $G$-invariant line in $\mathrm{H}\left(-w_{0} \mu\right) \otimes \mathrm{H}(\mu)$.

Proof. The first statement follows from Lemma 5.5. It follows by Frobenius reciprocity that $\mathrm{H}\left(-w_{0} \mu\right) \otimes \mathrm{H}(\mu)$ contains a unique $\Delta G$-invariant line. Hence, it suffices to show that $R_{\mu}\left(\operatorname{Tr}_{\mu}, 0\right)$ is nonzero. Consider the decomposition

$$
\operatorname{Tr}_{\mu}=\sum_{\theta \in X^{*}(T) \times X^{*}(T)}\left(\operatorname{Tr}_{\mu}\right)_{\theta}
$$

of $\operatorname{Tr}_{\mu}$ into $T \times T$-semiinvariant elements. Then $\left(\operatorname{Tr}_{\mu}\right)_{\left(-w_{0} \mu, w_{0} \mu\right)}$ is a nonzero multiple of $u_{\mu}^{+} \otimes v_{\mu}^{-}$, and the second statement now follows from the first statement. 


\section{LinE BUNDLES ON EQUIVARIANT EMBEDDINGS}

Throughout this section we assume that $G$ is simply connected. We use the notation introduced in Section 5. Let $X$ denote an equivariant embedding of $G$.

Lemma 6.1. Assume that $\psi_{\mu}$ extends to a map

$$
\psi_{\mu}: X \rightarrow \mathbb{P}_{\mu}
$$

and let $\tau_{\mu}$ denote the pull back of the global section $\left(u_{\mu}^{-} \otimes v_{\mu}^{-}, 0\right)$ of $\mathcal{O}_{\mu}(1)$. Then the support of the divisor of zeroes of $\tau_{\mu}$ does not contain any of the irreducible components of $X \backslash G$.

Proof. Notice first that the image $\psi_{\mu}(X)$ is contained in $Z_{\mu}$. If the support of the divisor of zeroes of $\tau_{\mu}$ contains an irreducible component of $X \backslash G$ then this support also contains a $G \times G$-orbit. Hence, also the support of the zero divisor of $\left(u_{\mu}^{-} \otimes v_{\mu}^{-}, 0\right)$ would contain a $G \times G$-orbit within $Z_{\mu}$. But the latter support is closed and hence it will contain the unique closed $G \times G$-orbit of $Z_{\mu}$ (see Lemma 5.3). In particular, $R_{\mu}\left(u_{\mu}^{-} \otimes v_{\mu}^{-}, 0\right)$, and hence also $R_{\mu}\left(u_{\mu}^{+} \otimes v_{\mu}^{-}, 0\right)$, is zero. By Lemma 5.6 this is a contradiction.

Let $D_{i}$ denote the closure of the Bruhat cell $B s_{i} w_{0} B$ within $X$. Then

Proposition 6.1. Assume that $\psi_{\omega_{i}}$ extends to a morphism

$$
\psi_{\omega_{i}}: X \rightarrow \mathbb{P}_{\omega_{i}}
$$

Then $D_{i}$ is a locally principal Weil divisor and its associated line bundle $\mathcal{O}_{X}\left(D_{i}\right)$ (resp. canonical section) is isomorphic to $\psi_{\omega_{i}}^{*}\left(\mathcal{O}_{\omega_{i}}(1)\right.$ ) (resp. $\left.\psi_{\omega_{i}}^{*}\left(u_{\omega_{i}}^{-} \otimes v_{\omega_{i}}^{-}, 0\right)\right)$.

Proof. By Lemma 5.1 we know that $\psi_{\omega_{i}, G}^{*}\left(u_{\omega_{i}}^{-} \otimes v_{\omega_{i}}^{-}, 0\right)$ is a generator of the lowest weight line in $\mathrm{H}\left(-w_{0} \omega_{i}\right)$. Let $\hat{i}$ denote the integer satisfying $\omega_{\hat{i}}=-w_{0} \omega_{i}$. Then $w_{0} s_{\hat{i}}=s_{i} w_{0}$ and the statement is hence an immediate consequence of Lemma 5.2 and Lemma 6.1

As $G$ is simply connected and as $X$ is normal any line bundle on $X$ will have a unique $G \times G$-linearization. This explains what is meant by an invariant global section in the following statement.

Corollary 6.1. Assume that $D_{i}$ is a locally principal Weil divisor on $X$. Then there exists a $G \times G$-equivariant morphism :

$$
\psi_{i}: \operatorname{End}\left(\mathrm{H}\left(\omega_{i}\right)\right)^{*} \oplus k \rightarrow \mathrm{H}^{0}\left(X, \mathcal{O}_{X}\left(D_{i}\right)\right),
$$

making the following diagram commutative

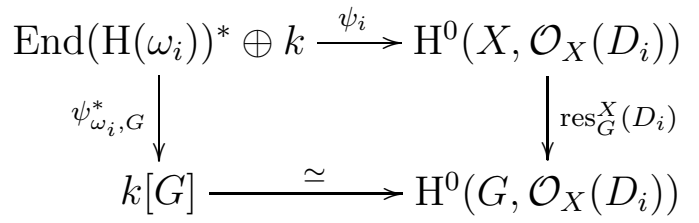


where the lower map is some (unique up to nonzero scalar) $G \times G$ equivariant identification. In particular, there exists a nonzero $G \times G$ invariant global section of $\mathcal{O}_{X}\left(D_{i}\right)$.

Proof. Consider the closure $\Gamma$ of the graph of $\psi_{\omega_{i}}$ within $X \times \mathbb{P}_{\omega_{i}}$. Projection on the first coordinate defines a birational projective morphism $\phi: \Gamma \rightarrow X$, which is an isomorphism outside a closed subset of $X$ of codimension $\geq 2$ (see e.g. Prop.III.9.1, [8]). In particular, there exists a $G \times G$-stable open subset $X^{\prime}$ of $X$ with $X \backslash X^{\prime}$ of codimension $\geq 2$ and an extension of $\psi_{\omega_{i}}$ to $X^{\prime}$.

As $X$ is normal $\mathrm{H}^{0}\left(X, \mathcal{O}_{X}\left(D_{i}\right)\right)=\mathrm{H}^{0}\left(X^{\prime}, \mathcal{O}_{X}\left(D_{i}\right)\right)$, which means that it suffices to prove the statement for $X^{\prime}$. As $\psi_{\omega_{i}}$ extends to $X^{\prime}$ the first assertion now follows from Proposition 6.1] To prove the second assertion it suffices to prove that $\psi_{i}(0,1)$ is nonzero. But this follows as $\psi_{\omega_{i}, G}^{*}(0,1)$ is a nonzero (constant) function on $G$.

\section{Frobenius SPLITTINGS OF SIMPLY CONNECTED GROUPS}

In this section $G$ denotes a simply connected group. The aim of this section is to construct a class of Frobenius splittings of $G$. These will be constructed by restricting Frobenius splittings of a certain equivariant embeddings $X$ of $G$. We begin by fixing the required properties of $X$.

Lemma 7.1. There exists a smooth complete toroidal embedding $X$ of $G$ such that the morphisms $\psi_{\omega_{i}}, i=1, \ldots, l$, all extend to $X$.

Proof. Start by choosing a complete toroidal embedding $X^{\prime}$ of $G$. Consider the product map $\psi:=\prod_{i=1}^{l} \psi_{\omega_{i}}: G \rightarrow \prod_{i=1}^{l} \mathbb{P}_{\omega_{i}}$, and let $\Gamma$ denote the normalization of the closure of the graph of $\psi$ within $X^{\prime} \times \prod_{i=1}^{l} \mathbb{P}_{\omega_{i}}$. Then any projective resolution (see Theorem 3.1) of $\Gamma$ has the desired properties.

Remark 2. A closer study of toroidal embeddings reveals that $\psi_{\omega_{i}}$ extends to arbitrary toroidal embedding. In particular, on a toroidal embedding of $G$ the Weil divisors $D_{i}$ are all locally principal.

For the rest of this section we will fix a toroidal embedding $X$ of $G$ satisfying the requirements in Lemma 7.1$]$ Fix a closed $G \times G$-orbit $Y$ within $X$. As noted in Section 3.2 we may $G \times G$-equivariantly identify $Y$ with the variety $G / B \times G / B$.

7.1. The canonical bundle. Let $X_{j}, j=1, \ldots, n$ denote the boundary components of $X$, i.e. the irreducible components of $X \backslash G$, all of codimension 1 as $G$ is affine (Chap.III, (4)). Let furthermore $D_{i}$ denote the closure of the Bruhat cell $B s_{i} w_{0} B$ within $X$. By Prop.6.2.6 of [3] it follows that the dual canonical bundle of $X$ is

$$
\omega_{X}^{-1} \simeq \mathcal{O}_{X}\left(\sum_{j=1}^{n} X_{j}+2 \sum_{i=1}^{l} D_{i}\right) .
$$


As we will see below, the restriction of the line bundle $\mathcal{O}_{X}\left(2 \sum_{i=1}^{l} D_{i}\right)$ to $Y$ is isomorphic to $\mathcal{L}_{G / B}(2 \rho) \otimes \mathcal{L}_{G / B}(2 \rho)$, which is the dual canonical bundle of $Y$. Let now $\sigma_{j}$ denote a canonical section of the line bundle $\mathcal{O}_{X}\left(X_{j}\right)$ and let $D$ denote the divisor $\sum_{i=1}^{l} D_{i}$. Then the following statement describes the usual way of constructing Frobenius splittings of $X$.

Theorem 7.1. Let $\tau$ denote a global section of $\mathcal{O}_{X}(2(p-1) D)$ such that its restriction $\tau_{\mid Y}$ to $Y$ corresponds to a Frobenius splitting of $Y$. Then the global section $\tau \prod_{j=1}^{n} \sigma_{j}^{p-1}$ of $\omega_{X}^{1-p}$ corresponds (up to a nonzero scalar) to a Frobenius splitting of $X$.

Proof. See e.g. proof of Thm.6.2.7 in [3].

7.2. Frobenius splittings of $X$. By the assumptions on $X$ there exists a commutative diagram of $G \times G$-equivariant morphisms

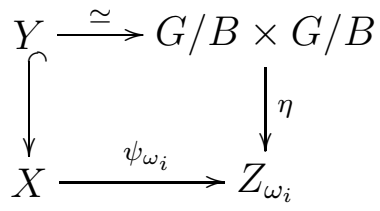

Hence, combining Proposition 6.1 and Lemma [5.5] we obtain

Proposition 7.2. There exists a commutative diagram

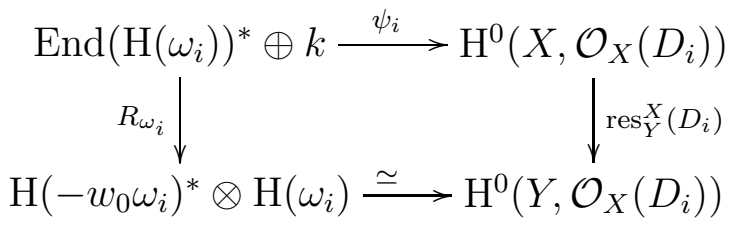

In particular, the image of the restriction map $\operatorname{res}_{Y}^{X}\left(D_{i}\right)$ contains the unique $B \times B$-stable line of $\mathrm{H}^{0}\left(Y, \mathcal{O}_{X}\left(D_{i}\right)\right)$.

It follows from Lemma 5.5 that the restriction of $\mathcal{O}_{X}((p-1) D)$ to $Y$ is isomorphic to the line bundle $\mathcal{L}_{G / B}((p-1) \rho) \otimes \mathcal{L}_{G / B}((p-1) \rho)$. This leads to the following statement.

Corollary 7.1. There exists a commutative diagram

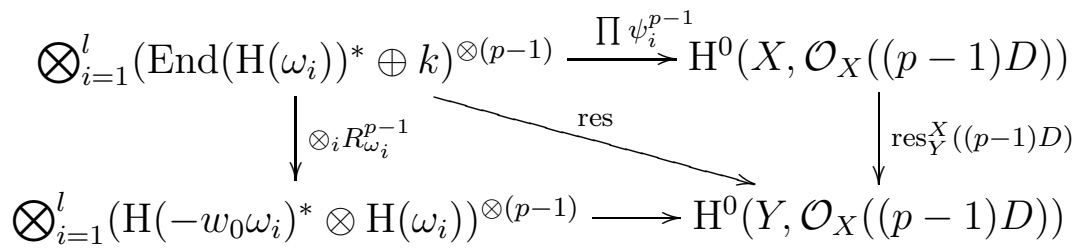

where the map res is surjective.

Proof. By Proposition 7.2 the image of res is nonzero. Hence the statement follows as $\mathrm{H}^{0}\left(Y, \mathcal{O}_{X}((p-1) D)\right) \simeq \mathrm{St} \otimes$ St is a simple $G \times G$ module 
Let $v_{-}$(resp. $\left.v_{+}\right)$denote a generator of the lowest (resp. highest) weight space in St and let $v_{\Delta}$ denote a generator of the unique diagonal $G$-invariant line in St $\otimes$ St. Applying Lemma 5.6] we find

Lemma 7.2. With $a_{1}, \ldots, a_{l} \in k$ we have

(1) $\operatorname{res}\left(\bigotimes_{i=1}^{l}\left(u_{\omega_{i}}^{+} \otimes v_{\omega_{i}}^{-}, 0\right)^{\otimes(p-1)}\right)$ is nonzero multiple of $v_{+} \otimes v_{-}$.

(2) $\operatorname{res}\left(\bigotimes_{i=1}^{l}\left(\operatorname{Tr}_{\omega i}, a_{i}\right)^{\otimes(p-1)}\right)$ is a nonzero multiple of $v_{\Delta}$.

By Theorem 7.1 and Corollary 4.1 we can now state and prove.

Theorem 7.3. Let $a_{1}, \ldots, a_{l} \in k$. Then the global section

$$
\prod_{i=1}^{l} \psi_{i}\left(\operatorname{Tr}_{\omega i}, a_{i}\right)^{p-1} \prod_{i=1}^{l} \psi_{i}\left(u_{\omega_{i}}^{+} \otimes v_{\omega_{i}}^{-}, 0\right)^{p-1} \prod_{j=1}^{n} \sigma_{j}^{p-1},
$$

of $\omega_{X}^{1-p}$ defines a Frobenius splitting of $X$ (up to a nonzero constant).

7.3. Frobenius splittings of $G$. The canonical bundle $\omega_{G}$ of $G$ is trivial and we may therefore choose a volume form $d G$ freely generating the global sections of $\omega_{G}$ as a $k[G]$-module. Restricting the statement in Theorem $[7.3$ to the open subset $G$ then implies

Corollary 7.2. Let $a_{1}, \ldots, a_{l} \in k$. Then the global section

$$
\prod_{i=1}^{l} \psi_{\omega_{i}, G}^{*}\left(\operatorname{Tr}_{\omega i}, a_{i}\right)^{p-1} \prod_{i=1}^{l} \psi_{\omega_{i}, G}^{*}\left(u_{\omega_{i}}^{+} \otimes v_{\omega_{i}}^{-}, 0\right)^{p-1} d G^{1-p}
$$

of $\omega_{G}^{1-p}$ defines a Frobenius splitting of $X$ (up to a nonzero constant).

\section{Frobenius splitting in the general CASE}

In this section $G$ denotes a simply connected linear algebraic group. Let $X$ denote an equivariant embedding of $G$ and let $D_{i}\left(\operatorname{resp} . \tilde{D}_{i}\right)$ denote the closure of the Bruhat cell $B s_{i} w_{0} B$ (resp. $B^{+} s_{i} B$ ) within X. As in the previous section we let $X_{j}, j=1, \ldots, n$, denote the irreducible components of $X \backslash G$. Moreover when $\mathbf{a} \in \mathbb{A}^{l}$, we let $\bar{\chi}^{-1}(\mathbf{a})$ denote the closure of the associated Steinberg fibre within $X$.

8.1. The smooth case. Assume first that $X$ is smooth. In this case the divisors $X_{j}$ are all locally principal and we choose canonical sections $\sigma_{j}$ of the associated line bundles $\mathcal{O}_{X}\left(X_{i}\right)$. By Prop.6.2.6. in [3] the dual canonical bundle of $X$ is isomorphic to

$$
\omega_{X}^{-1} \simeq \mathcal{O}_{X}\left(\sum_{j=1}^{n} X_{j}+2 \sum_{i=1}^{l} D_{i}\right)
$$

In order to construct Frobenius splittings of $X$ we have to construct global sections of $\omega_{X}^{1-p}$. First we apply Corollary 6.1 and obtain for each $i=1, \ldots, l$, a commutative diagram of $G \times G$-equivariant maps : 


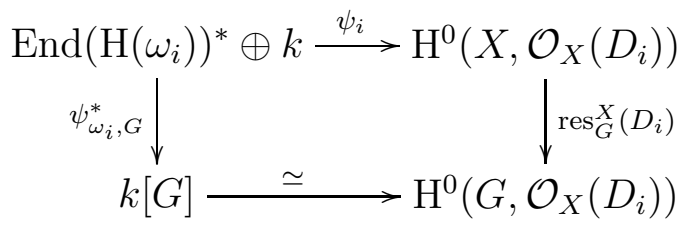

Now we have the following generalization of Theorem $[7.3$

Corollary 8.1. Let $\mathbf{a}=\left(a_{1}, \ldots, a_{l}\right) \in \mathbb{A}^{l}$. Then the global section

$$
\tau_{\mathbf{a}}=\prod_{i=1}^{l} \psi_{i}\left(\operatorname{Tr}_{\omega i},-a_{i}\right)^{p-1} \prod_{i=1}^{l} \psi_{i}\left(u_{\omega_{i}}^{+} \otimes v_{\omega_{i}}^{-}, 0\right)^{p-1} \prod_{j=1}^{n} \sigma_{j}^{p-1}
$$

of $\omega_{X}^{1-p}$ defines a Frobenius splitting of $X$ (up to a nonzero constant) which compatibly splits the closed subvarieties $\bar{\chi}^{-1}(\mathbf{a}), \tilde{D}_{i}, i=1, \ldots, l$, and $X_{j}, j=1, \ldots, n$.

Proof. By Corollary 7.2 the restriction of $\tau_{\mathbf{a}}$ to $G$ defines a Frobenius splitting of $G$ and hence, by Remark 1, $\tau_{\mathbf{a}}$ defines a Frobenius splitting of $X$. By Lemma 4.10 and Lemma 4.5 each component of the zero divisor of $\tau_{\mathbf{a}}$ is compatibly Frobenius split. In particular, the boundary divisors $X_{j}$ and (by Proposition 6.1) the subvarieties $\tilde{D}_{i}$ are all compatibly Frobenius split. Finally, by Lemma 4.5, every component of the (scheme theoretic) intersection of the zero divisors of $\psi_{i}\left(\operatorname{Tr}_{\omega i},-a_{i}\right)$ will be Frobenius split. But, by Lemma 5.1 the component of this latter intersection which intersects $G$ nontrivially is exactly the subvariety $\bar{\chi}^{-1}(\mathbf{a})$.

The following connected result will also be useful.

Proposition 8.1. Let $\mathbf{a}=\left(a_{1}, \ldots, a_{l}\right) \in \mathbb{A}^{l}$. Then there exists a stable Frobenius splitting of $X$ along the divisor

$$
(p-1)\left(\sum_{j=1}^{n} X_{j}+\sum_{i=1}^{l} \tilde{D}_{i}\right)
$$

of degree 1 which compatibly Frobenius splits the subvariety $\bar{\chi}^{-1}(\mathbf{a})$.

Proof. By Lemma 4.10 and Lemma 4.6 the Frobenius splitting $\tau_{\mathbf{a}}$ in Corollary 8.1 defines a degree 1 stable Frobenius splitting $s$ of $X$ along the divisor

$$
(p-1)\left(\sum_{j=1}^{n} X_{j}+\sum_{i=1}^{l} \tilde{D}_{i}\right),
$$

which compatibly Frobenius splits the zero divisor of the global section

$$
\prod_{i=1}^{l} \psi_{i}\left(\operatorname{Tr}_{\omega i},-a_{i}\right)
$$


of the line bundle $\mathcal{O}_{X}\left(\sum_{i=1}^{l} D_{i}\right)$. Denote by $s^{\prime}$ the (by $s$ ) induced degree 1 stable Frobenius splitting of $X$ along the zero divisor 0. Combining Lemma 4.3 and Lemma 4.5 and arguing as in the last part of the proof of Corollary 8.1. we find that the closed subvariety $\bar{\chi}^{-1}(\mathbf{a})$ is compatibly Frobenius split by $s^{\prime}$. By Lemma 4.2 it remains to prove that $\bar{\chi}^{-1}(\mathbf{a})$ is not contained in the support of the divisor $\sum_{j=1}^{n} X_{j}+\sum_{i=1}^{l} \tilde{D}_{i}$. This follows from Lemma 8.1 below.

Lemma 8.1. The closure of the subset $B^{+} s_{i} B$ within $G$ does not contain any B-conjugacy classes.

Proof. Let $f$ denote a generator of the highest weight line in $\mathrm{H}\left(\omega_{i}\right)$. By Lemma 5.2 the zero set of $f$ coincides with the closure of $B^{+} s_{i} B$. So assume that there exists an element $g \in G$ such that $f\left(b g b^{-1}\right)=0$ for all $b \in B$. By definition of $\mathrm{H}\left(\omega_{i}\right)$ this implies that $f(b g)=0$ for all $b \in B$. Moreover, as $f$ is $B^{+}$-semiinvariant we find that $f(h g)=0$ for every element $h$ in the open dense subset $B^{+} B$ of $G$. In particular, $f$ vanishes on an open dense subset of $G$ which is a contradiction.

Corollary 8.2. Assume that $X$ is projective and let $\mathbf{a}=\left(a_{1}, \ldots, a_{l}\right) \in$ $\mathbb{A}^{l}$. Then there exists a stable Frobenius splitting of $X$ along an ample divisor with support $X \backslash G$ which compatibly Frobenius splits the subvariety $\bar{\chi}^{-1}(\mathbf{a})$.

Proof. By Proposition 8.1 and Lemma 4.3 there exists a stable Frobenius splitting of $X$ along the divisor $\sum_{j=1}^{n} X_{j}$ which compatibly splits $\bar{\chi}^{-1}(\mathbf{a})$. Applying Lemma 4.3 and Lemma 4.4 it suffices to show that there exists positive integers $c_{j}>0$ such that $\sum_{j=1}^{n} c_{j} X_{j}$ is ample. This follows from Prop.4.1(2) in [2].

8.2. The general case. For a general equivariant $G$-embedding we can now prove.

Theorem 8.2. Let $X$ denote an arbitrary equivariant $G$-embedding and let $\mathbf{a}=\left(a_{1}, \ldots, a_{l}\right) \in \mathbb{A}^{l}$. Then $X$ is Frobenius splits compatibly with the closed subvarieties $\bar{\chi}^{-1}(\mathbf{a}), \tilde{D}_{i}, i=1, \ldots, l$ and $X_{j}, j=$ $1, \ldots, n$.

Proof. By Theorem 3.1 there exists a resolution $f: X^{\prime} \rightarrow X$ of $X$ by a smooth $G$-embedding $X^{\prime}$. By Zariski's main theorem we know $f_{*} \mathcal{O}_{X^{\prime}} \simeq \mathcal{O}_{X}$. Hence, by the discussion in Section 4.5 it suffices to prove the above statement for $X^{\prime}$. Now apply Corollary 8.1 .

8.3. Canonical Frobenius splittings. Let $Z$ denote an arbitrary $G$ variety and let $s: F_{*} \mathcal{O}_{Z} \rightarrow \mathcal{O}_{Z}$ denote a Frobenius splitting of $Z$. For any root $\alpha$ we let $x_{\alpha}: k \rightarrow G$ denote the associated root homomorphism satisfying $t \in T, c \in k: t x_{\alpha}(c) t^{-1}=x_{\alpha}(\alpha(t) c)$. Recall (Defn.4.1.1, [3] ) 
that $s$ is said to be a canonical Frobenius splitting if $s$ is $T$-invariant and satisfies

$$
x_{\alpha_{i}}(c) s=\sum_{j=1}^{p-1} c^{j} s_{j}
$$

for every simple root $\alpha_{i}$, and certain elements $s_{j} \in \operatorname{Hom}_{\mathcal{O}_{X}}\left(F_{*} \mathcal{O}_{Z}, \mathcal{O}_{Z}\right)$. The primary reason for the interest in canonical Frobenius splittings comes from the following consequence (see e.g. Thm.4.2.13 in [3])

Theorem 8.3. Assume that $Z$ has a canonical Frobenius splitting. Let $\mathcal{L}$ denote a $G$-linearized line bundle on $Z$. Then the $G$-module $\mathrm{H}^{0}(Z, \mathcal{L})$ admits a good filtration, i.e. there exists a sequence of $G$-modules

$$
0=M^{0} \subseteq M^{1} \subseteq M^{2} \subseteq \cdots
$$

such that $\mathrm{H}^{0}(Z, \mathcal{L})=\cup_{i} M^{i}$ and satisfying that the successive quotients $M^{j+1} / M^{j}$ are isomorphic to modules of the form $\mathrm{H}\left(\lambda_{j}\right)$ for certain dominant weights $\lambda_{j}$.

Let $\mathbf{a}=\left(a_{1}, \ldots, a_{l}\right) \in \mathbb{A}^{l}$ and consider an arbitrary equivariant embedding $X$ of $G$. Then there exists a diagonal $G$-action on the closed subvariety $\bar{\chi}^{-1}(\mathbf{a})$. We claim

Corollary 8.3. The variety $\bar{\chi}^{-1}(\mathbf{a})$ admits a canonical Frobenius splitting. In particular, when $\mathcal{L}$ is a $G$-linearized line bundle on $\bar{\chi}^{-1}(\mathbf{a})$ then the $G$-module $\mathrm{H}^{0}\left(\bar{\chi}^{-1}(\mathbf{a}), \mathcal{L}\right)$ has a good filtration.

Proof. By the results in the previous section $\bar{\chi}^{-1}(\mathbf{a})$ is compatibly Frobenius split by a Frobenius splitting $s$ of $X$. Hence, it suffices to prove that $s$ is a canonical Frobenius splitting of $X$. By the proof of Theorem 8.2 it moreover suffices to consider the case when $X$ is smooth and $s$ is defined from $\tau_{\mathbf{a}}$ (with notation as in Corollary 8.1). Now every factor of $\tau_{\mathbf{a}}$ except

$$
\prod_{i=1}^{l} \psi_{i}\left(u_{\omega_{i}}^{+} \otimes v_{\omega_{i}}^{-}, 0\right)^{p-1}
$$

is invariant under the diagonal $G$-action. Hence we may concentrate on the (diagonal) $T$-invariant factor $\psi_{i}\left(u_{\omega_{i}}^{+} \otimes v_{\omega_{i}}^{-}, 0\right)$. The statement now follows as, for all $j$,

$$
\begin{gathered}
x_{\alpha_{j}}(c) v_{\omega_{i}}^{-}=v_{\omega_{i}}^{-}+c v_{i, j}, \\
x_{\alpha_{j}}(c) u_{\omega_{i}}^{+}=u_{\omega_{i}}^{+},
\end{gathered}
$$

for certain elements $v_{i, j} \in \mathrm{H}\left(\omega_{i}\right)$ (recall that the $\left(w_{0} \omega_{i}+q \alpha_{j}\right)$-weight space of $\mathrm{H}\left(\omega_{i}\right)$ is zero for $\left.q>1\right)$.

\section{Cohomology vanishing}

In this section we will discuss various results which will enable us to prove that closures of Steinberg fibers in arbitrary equivariant embeddings have nice geometric properties 
9.1. Resolutions and direct images. Let $X^{\prime}$ denote a projective equivariant embedding of $G$ and let $f: X \rightarrow X^{\prime}$ be a resolution of $X^{\prime}$ by a smooth projective equivariant embedding. When $\mathbf{a}=\left(a_{1}, \ldots, a_{l}\right) \in$ $\mathbb{A}^{l}$ we denote by $\bar{\chi}^{-1}(\mathbf{a})$ (resp. $\left.\bar{\chi}^{-1}(\mathbf{a})^{\prime}\right)$ the closure of the Steinberg fiber at a within $X$ (resp. $\left.X^{\prime}\right)$.

Corollary 9.1. With the notation described above we have

(1) $f_{*} \mathcal{O}_{X}=\mathcal{O}_{X^{\prime}}$ and $R^{i} f_{*} \mathcal{O}_{X}=0$ when $i>0$.

(2) $f_{*} \mathcal{O}_{\bar{\chi}^{-1}(\mathbf{a})}=\mathcal{O}_{\bar{\chi}^{-1}(\mathbf{a})^{\prime}}$ and $R^{i} f_{*} \mathcal{O}_{\bar{\chi}^{-1}(\mathbf{a})}=0$ when $i>0$.

Proof. As $X^{\prime}$ is normal and $f$ is birational it follows from Zariski's main theorem that $f_{*} \mathcal{O}_{X}=\mathcal{O}_{X^{\prime}}$. Hence, by Lemma 4.9 it suffices to prove that there exists a stable Frobenius splitting of $X$ along an ample divisor which compatibly Frobenius splits $\bar{\chi}^{-1}(\mathbf{a})$. Now apply Corollary 8.2 .

9.2. Cohomology. We are now ready to prove the following statement about cohomology of line bundles on closures of Steinberg fibers.

Proposition 9.1. Let $X$ denote a projective equivariant embedding of $G$ and let $\mathbf{a}=\left(a_{1}, \ldots, a_{l}\right) \in \mathbb{A}^{l}$. Let $\mathcal{M}$ (resp. $\left.\mathcal{L}\right)$ denote a globally generated line bundle on $X$ (resp. $\bar{\chi}^{-1}(\mathbf{a})$ ). Then

$$
\mathrm{H}^{i}(X, \mathcal{M})=\mathrm{H}^{i}\left(\bar{\chi}^{-1}(\mathbf{a}), \mathcal{L}\right)=0, i>0 .
$$

Moreover, the restriction map

$$
\mathrm{H}^{0}(X, \mathcal{L}) \rightarrow \mathrm{H}^{0}\left(\bar{\chi}^{-1}(\mathbf{a}), \mathcal{L}\right)
$$

is surjective.

Proof. By Corollary 9.1 we may assume that $X$ is smooth. Now apply Corollary 8.2 and the "in particular" parts of Lemma 4.7 and Lemma 4.8.

\section{Geometry of Closures of Steinberg Fibers}

In this section we will study the geometry of the closure of a Steinberg fiber within an equivariant $G$-embedding $X$ of a simply connected group $G$.

10.1. The smooth case. Assume that $X$ is a smooth $G$-embedding and let $\mathbf{a}=\left(a_{1}, \ldots, a_{l}\right) \in \mathbb{A}^{l}$. Let $\mathcal{O}_{\mathbf{a}}$ denote the restriction of the line bundle

$$
\mathcal{O}_{X}\left(\sum_{j=1}^{n} X_{j}+\sum_{i=1}^{l} \tilde{D}_{i}\right)
$$

to $\bar{\chi}^{-1}(\mathbf{a})$ and and let $s_{\mathbf{a}}$ denote the associated restricted canonical section. Notice that by Proposition 8.1 the section $s_{\mathbf{a}}$ is nonzero. 
Theorem 10.1. Let $X$ denote a smooth $G$-embedding and let $\mathbf{a}=$ $\left(a_{1}, \ldots, a_{l}\right) \in \mathbb{A}^{l}$. Then the closure $\bar{\chi}^{-1}(\mathbf{a})$ of the Steinberg fiber $\chi^{-1}(\mathbf{a})$ within $X$ is a local complete intersection. Moreover, the dualizing sheaf on $\bar{\chi}^{-1}(\mathbf{a})$ equals the line bundle $\mathcal{O}_{\mathbf{a}}^{-1}$.

Proof. As $\omega_{X}^{-1}=\mathcal{O}_{X}\left(\sum_{j} X_{j}+\sum_{i}\left(D_{i}+\tilde{D}_{i}\right)\right)$ it suffices to prove that $\bar{\chi}^{-1}(\mathbf{a})$ coincides with the intersection $Z$ of the zero schemes of the sections $\psi_{i}\left(\operatorname{Tr}_{\omega i},-a_{i}\right), i=1, \ldots, l$. When $X=G$ this is clearly the case. Hence, it suffices to prove that all components of $Z$ intersect the open subset $G$. So assume that $Z^{\prime}$ is a component of $Z$ which does not intersect $G$. Then $Z^{\prime}$ must be contained in one of the boundary components $X_{j}$. Choose an open affine subset $U$ such that $\emptyset \neq U \cap Z^{\prime}=$ $U \cap Z$ and such that the canonical bundle $\omega_{U}$ is trivial. Choose also a volume form $d U$, i.e. a global section of $\omega_{U}$ generating the set of global sections as a $k[U]$-module. Then $\tau_{\mathbf{a}}$ (with notation as in Corollary 8.1) restricts to a Frobenius splitting of $U$ of the form

$$
\left(\tau_{\mathbf{a}}\right)_{\mid U}=\left(\prod_{i=1}^{l} f_{i}^{p-1} \prod_{i=1}^{l} g_{i}^{p-1} \prod_{j=1}^{n} h_{j}^{p-1}\right) \cdot(d U)^{1-p}
$$

where $f_{i}, g_{i}$ and $h_{i}$ are the functions on $U$ defining the restrictions of the zero sets of $\psi_{i}\left(\operatorname{Tr}_{\omega i},-a_{i}\right), \psi_{i}\left(u_{\omega_{i}}^{+} \otimes v_{\omega_{i}}^{-}, 0\right)$ and $\sigma_{j}$ respectively. By assumption, the common zero $V\left(f_{1}, \ldots, f_{l}\right)$ of $f_{1}, \ldots, f_{l}$ is contained in the zero set $V\left(h_{j}\right)$ of $h_{j}$. Hence by Hilberts Nullstellensatz, $h_{j}$ is contained in the radical of the ideal $\left(f_{1}, \ldots, f_{l}\right)$. But $\left(f_{1}, \ldots, f_{l}\right)$ is compatibly split by Lemma 4.10 and Lemma 4.5 and therefore it is radical by Lemma 4.5. We conclude, that $h_{j} \in\left(f_{1}, \ldots, f_{l}\right)$ and hence

$$
\prod_{i=1}^{l} f_{i}^{p-1} \prod_{i=1}^{l} g_{i}^{p-1} \prod_{j=1}^{n} h_{j}^{p-1} \in\left(f_{1}^{p}, \ldots, f_{l}^{p}\right) .
$$

In particular, the Frobenius splitting $\left(\tau_{\mathbf{a}}\right)_{\mid U}$ of $U$ will map the constant function 1 to an element within the ideal $\left(f_{1}, \ldots, f_{l}\right)$. Therefore $1 \in$ $\left(f_{1}, \ldots, f_{l}\right)$ and hence

$$
Z^{\prime} \cap U=V\left(f_{1}, \ldots, f_{l}\right)=\emptyset,
$$

which is a contradiction.

Corollary 10.1. Let $X$ denote a smooth $G$-embedding and let $\mathbf{a}=$ $\left(a_{1}, \ldots, a_{l}\right) \in \mathbb{A}^{l}$. Then the closure $\bar{\chi}^{-1}(\mathbf{a})$ of the Steinberg fiber $\chi^{-1}(\mathbf{a})$ within $X$ is normal, Gorenstein and Cohen-Macaulay.

Proof. By Theorem 10.1 it suffices to show that the closure $\bar{\chi}^{-1}(\mathbf{a})$ is smooth in codimension 1 . So let $Z$ denote an irreducible component of the singular locus of $\bar{\chi}^{-1}(\mathbf{a})$. If $G \cap Z \neq \emptyset$ then the codimension of $Z$ is $\geq 2$ as $\chi^{-1}(\mathbf{a})$ is normal by Thm.6.11 in 11. So assume that $Z$ is contained in one of the boundary components $X_{j}$ of $X$. 
Consider the scheme theoretic intersection $X_{j} \cap \bar{\chi}^{-1}(\mathbf{a})$ which is reduced by Corollary 8.1 and Lemma 4.5. Hence, as $X_{j}$ is locally principal every smooth point of $X_{j} \cap \bar{\chi}^{-1}(\mathbf{a})$ will also be a smooth point of $\bar{\chi}^{-1}(\mathbf{a})$. In particular, $Z$ is properly contained in one of the components of $X_{j} \cap \bar{\chi}^{-1}(\mathbf{a})$. But the components of the latter scheme all have codimension 1 in $\bar{\chi}^{-1}(\mathbf{a})$ and hence $Z$ must have codimension $\geq 2$ in $\bar{\chi}^{-1}(\mathbf{a})$. This ends the proof.

10.2. General $G$-embeddings. Now assume that $X$ is an arbitrary equivariant embedding of a simply connected group $G$. The following result is due to G. Kempf. The version stated here is taken from [1].

Lemma 10.1. Let $f: Z^{\prime} \rightarrow Z$ denote a proper map of algebraic schemes satisfying that $f_{*} \mathcal{O}_{Z^{\prime}}=\mathcal{O}_{Z}$ and $R^{i} f_{*} \mathcal{O}_{Z^{\prime}}=0, i>0$. If $Z^{\prime}$ is Cohen-Macaulay with dualizing sheaf $\omega_{Z^{\prime}}$ and if $R^{i} f_{*} \omega_{Z^{\prime}}=0$ for $i>0$, then $Z$ is Cohen-Macaulay with dualizing sheaf $f_{*} \omega_{Z^{\prime}}$.

We will also need the following result due to Mehta and van der Kallen $([7])$

Lemma 10.2. Let $f: Z^{\prime} \rightarrow Z$ denote a proper morphism of schemes and let $V^{\prime}$ (resp. $V$ ) denote a closed subscheme of $Z^{\prime}$ (resp. Z). Let $\mathcal{I}_{V^{\prime}}$ denote the sheaf of ideals of $V^{\prime}$. Fix an integer $i$ and assume

(1) $f^{-1}(V) \subseteq V^{\prime}$.

(2) $R^{i} f_{*} \mathcal{I}_{V^{\prime}}$ vanishes outside $V$.

(3) $V^{\prime}$ is compatibly Frobenius split in $Z^{\prime}$.

Then $R^{i} f_{*} \mathcal{I}_{V^{\prime}}=0$.

We are ready to prove

Theorem 10.2. Let $X$ denote an arbitrary equivariant embedding of $G$ and let $\mathbf{a}=\left(a_{1}, \ldots, a_{l}\right) \in \mathbb{A}^{l}$. Then the closure $\bar{\chi}^{-1}(\mathbf{a})$ of the Steinberg fiber at $\mathbf{a}$ in $X$ is normal and Cohen-Macaulay.

Proof. Any equivariant embedding has an open cover by open equivariant subsets of projective equivariant embeddings. (see e.g. proof of Cor.6.2.8 in 3]) This reduces the statement to the case where $X$ is projective. Choose a projective resolution $f: X^{\prime} \rightarrow X$ of $X$ by a smooth equivariant embedding $X^{\prime}$. Then $f_{*} \mathcal{O}_{\bar{\chi}^{-1}(\mathbf{a})^{\prime}}=\mathcal{O}_{\bar{\chi}^{-1}(\mathbf{a})}$ and $R^{i} f_{*} \mathcal{O}_{\bar{\chi}^{-1}(\mathbf{a})^{\prime}}=0, i>0$, by Corollary 9.1 . Hence by Corollary 10.1 this implies that $\bar{\chi}^{-1}(\mathbf{a})$ is normal.

In order to show that $\bar{\chi}^{-1}(\mathbf{a})$ is Cohen-Macaulay we apply the above Lemma 10.1 and Lemma 10.2. By Theorem 10.1 it suffices to prove that $R^{i} f_{*} \mathcal{O}_{\mathbf{a}}^{\prime-1}=0, i>0$. Let $Z$ denote the closed subscheme of $\bar{\chi}^{-1}(\mathbf{a})^{\prime}$ defined by $s_{\mathbf{a}}^{\prime}$. Combining Proposition 8.1 and Lemma 4.6 we find that $\bar{\chi}^{-1}(\mathbf{a})^{\prime}$ is Frobenius split compatibly with the closed subscheme $Z$. Moreover, $f: \bar{\chi}^{-1}(\mathbf{a})^{\prime} \rightarrow \bar{\chi}^{-1}(\mathbf{a})$ is an isomorphism above the the open subset $\chi^{-1}(\mathbf{a})$ and

$$
f^{-1}\left(\bar{\chi}^{-1}(\mathbf{a}) \backslash \chi^{-1}(\mathbf{a})\right) \subseteq Z
$$


Hence, by Lemma 10.2 we conclude $R^{i} f_{*} \mathcal{I}_{Z}=0$ for $i>0$. But $\mathcal{I}_{Z} \simeq$ $\mathcal{O}^{\prime-1}$ ahich ends the proof.

Remark 3. Let $\bar{\chi}^{-1}(\mathbf{a})$ denote the closure of the Steinberg fiber at a point $\mathbf{a} \in \mathbb{A}^{l}$ within some equivariant embedding $X$ of $G$. Similar as to the situation for large Schubert varieties (see 2]) we expect that $\bar{\chi}^{-1}(\mathbf{a})$ is strongly F-regular (see [10]). In order to prove this it suffices to prove that $\bar{\chi}^{-1}(\mathbf{a})$ is globally F-regular (see [10]) when $X$ is a projective smooth embedding. In this case we have seen that $\bar{\chi}^{-1}(\mathbf{a})$ is compatibly Frobenius split by a stable Frobenius splitting of $X$ along an ample divisor $D$ with support $X \backslash G$ (Corollary 8.2). By Thm.3.10 in [10] it therefore suffices to prove that $\chi^{-1}(\mathbf{a})$ is strongly $F$-regular. When $\chi^{-1}(\mathbf{a})$ coincides with the conjugacy class of a regular semisimple element of $G$ this is clearly the case. However, for the most interesting fiber of $\chi$, i.e. the unipotent variety $\mathcal{U}$ of $G$, we do not know how to prove the latter statement.

\section{REFERENCES}

1. M. Brion and P. Polo, Large Schubert varieties, Represent. Theory 4 (2000), $97-126$.

2. M. Brion and J.F. Thomsen, F-regularity of large Schubert varieties, math.AG/0408180

3. M. Brion and S. Kumar, Frobenius splitting methods in geometry and representation theory, Progress in Math., Birkhäuser 2005.

4. R. Hartshorne, Ample subvarieties of algebraic varieties, Lecture Notes in Math. 156 (1970), Springer-Verlag.

5. N. Lauritzen and J.F. Thomsen, Frobenius splitting and hyperplane sections of flag manifolds, Invent. Math. 128 (1997), 437-442.

6. V. Mehta and A. Ramanathan, Frobenius splitting and cohomology vanishing for Schubert varieties, Ann. Math. 122 (1985), 27-40.

7. V. Mehta and van der Kallen, W. On a Grauert-Riemenschneider vanishing theorem for Frobenius split varieties in characteristic p, Invent. Math., 108 (1992), 11-13.

8. D. Mumford, The red book of varieties and schemes, first edition, SpringerVerlag, Berlin, 1988.

9. A. Ramanathan, Equations defining Schubert varieties and Frobenius splitting of diagonals, Pub. Math. IHES 65 (1987), 61-90.

10. K. E. Smith, Globally F-regular varieties: Applications to vanishing theorems for quotients of Fano varieties, Michigan Math. J. 48 (2000), 553-572.

11. R. Steinberg, Regular elements of semisimple algebraic groups, Inst. Hautes Étude Sci. Publ. Math. 25 (1965), 49-80.

Institut for matematiske fag, Aarhus Universitet, 8000 Århus C, DENMARK

E-mail address: funch@imf.au.dk 